\title{
EDITORIAL
}

\section{EDITORIAL: VIVIR LA CIUDAD: DINÁMICAS Y PROCESOS URBANOS $Y$ METROPOLITANOS EN CIUDADES ESPAÑOLAS}

\author{
Living the city: urban and metropolitan dynamics and processes in Spanish cities \\ Raúl Lardiés-Bosque \\ Dpto. de Geografía y Ordenación del Territorio \\ Escuela Politécnica Superior de Huesca (EPSH) \\ Universidad de Zaragoza \\ rlardies@unizar.es \\ ORCID iD: https://orcid.org/0000-0001-9912-0739 \\ Fermina Rojo-Pérez \\ Instituto de Economía, Geografía y Demografía (IEGD); Consejo Superior de Investigaciones Científicas (CSIC). Grupo de \\ Investigación sobre Envejecimiento (GIE-CSIC). Red de Envejecimiento de la Asociación Latinoamericana de Población \\ (ALAP). Madrid, España \\ fermina.rojo@csic.es \\ ORCID iD: https://orcid.org/0000-0001-9935-2548
}

Vivimos en un mundo cada vez más urbano, y España está inserta también en este proceso. Según datos de la Estadística del Padrón Continuo, el 79,7\% de alrededor de 47,3 millones de habitantes en España residía en municipios urbanos (>10.000 hab.) en 2020 y un $39,9 \%$ lo hacía en los de más de 100.000 habitantes. Es mucha población en poco territorio, por lo que las dinámicas y procesos sociodemográficos que se producen en estos espacios han sido y son de interés geodemográfico.

Las ciudades pueden concebirse como mosaicos de varias piezas, y en ellas participan distintos grupos y colectivos de población, tanto en sus zonas centrales como periféricas y/o metropolitanas. Como organismos vivos que son, en las ciudades se producen transformaciones sociodemográficas, nuevas dinámicas y procesos urbanos, motivados por diversas causas y con múltiples consecuencias. Las partes del mosaico van cambiando, se transforman, se modifica la estructura de su población y se extienden: algunas se vacían o repueblan debido a la llegada o la salida de grupos de población de distintas características, y, a la vez, se revalorizan o también se desvalorizan; algunas se rehabilitan, y unos grupos sustituyen a otros. Por ejemplo, antes de 2008 fueron muy analizados los procesos de suburbanización, pero la llegada de nueva población por inmigración así como los cambios económicos y la movilidad residencial, impulsaron importantes transformaciones desde ese momento.

Al interés inicial por los espacios urbanos centrales en las grandes ciudades españolas, le siguió la expansión residencial en las coronas metropolitanas, mientras que desde aproximadamente el año 2010 vuelve el atractivo de los espacios centrales (López Gay, 2016). No obstante, hay matices, ya que mientras en las grandes ciudades se está atravesando una fase de recentralización, en las pequeñas se alude a un proceso de concentración (Torrado, Duque-Calvache y Palomares-Linares, 2020). Este patrón de resurgimiento de "lo central", común en muchas ciudades occidentales (Torrado, Calvache y Susino, 2020), está vinculado, por un lado, a la localización de nuevos sectores productivos y a la presencia de enclaves culturales, creativos y de innovación. Por otro lado, ese resurgimiento de "lo central" se asocia también con el incipiente cambio en el sistema de tenencia del mercado de vivienda, con un creciente peso del alquiler, sobre todo en las edades adultas más jóvenes (Módenes y López-Colás, 2014). Entre las causas estructurales que apuntalan este interés actual por lo central destacan desde cambios en las preferencias residenciales, asociados a la segunda transición demográfica, hasta modificaciones de tipo estructural en la economía mundial, pasando por procesos socioespacia- 
les de gentrificación y renovación urbanas (Torrado, Calvache y Susino, 2020).

En paralelo, el patrón territorial de la movilidad residencial ha experimentado transformaciones muy notables en los últimos años, básicamente como consecuencia de la suburbanización, metropolización y dispersión de la ciudad.

Los límites físicos de las ciudades se modifican y las áreas metropolitanas se amplían y avanzan hacia la madurez tras décadas de crecimiento urbano y demográfico (López-Gay, 2016). La explotación de los Censos de Población y Viviendas y del Padrón continuo, a escala de barrio o inferiores, son las principales herramientas que permiten analizan esas transformaciones.

En esta sección monográfica se presenta una selección de aportaciones realizadas al XVII Congreso de la Población: "Despoblación, Dinámicas Demográficas y Vulnerabilidad Social", organizado por el Grupo de Población de la Asociación Española de Geografía (AGE), el Departamento de Geografía y Ordenación del Territorio de la Universidad de Zaragoza, y el Grupo de Investigación GEOT (Grupo de Estudios en Ordenación del Territorio, UZ), y celebrado en Zaragoza-Los Monegros entre el 10 y el 11 de junio de 2021. En concreto, la selección se ha realizado entre las aportaciones presentadas a la ponencia 2: Dinámicas Socioespaciales y Cambios Poblacionales en Áreas Urbanas: una Mirada pre y post Pandémica, coordinada por el Dr. Antonio López Gay, de la Universidad Autónoma de Barcelona y del Centro de Estudios Demográficos (CED).

Los siete trabajos publicados en esta sección, en su mayoría realizados por geógrafos, pero también por otros profesionales de disciplinas afines, analizan fenómenos y procesos producidos en ciudades grandes e intermedias, que son ligeramente diferentes de los ocurridos en las más pequeñas (González-Leonardo, 2020), al menos en su intensidad. En ellos se analizan cuestiones geodemográficas, de las dinámicas espaciales y temporales, de las características y de los fenómenos demográficos -como segregación o cambios en los hogares-, residenciales y migratorios, en algunas ciudades españolas. La inmigración, la elevada movilidad diaria y los cambios residenciales, la llegada de turistas y el problema con la vivienda, o el elevado número de hogares con personas mayores, son aspectos que acaparan atención, aunque también algunos autores realizan propuestas metodológicas para definir y caracterizar las áreas metropolitanas. Barcelona es el ámbito urbano y metropolitano que más trabajos concentra -hasta tres-, seguida por Ma- drid, Valencia, Las Palmas de Gran Canaria, Santander, y la región de Castilla y León.

De entre las aportaciones, un primer grupo de cuatro artículos se centra en distintos aspectos de la movilidad espacial de la población, analizando cuestiones de movilidad diaria y residencial de la población para caracterizar un área metropolitana (Olga de Cos Guerra), de cambios residenciales y migratorios de la población extranjera (Julia Salom-Carrasco), de la influencia de las migraciones en el metabolismo demográfico (Andreu Domingo y Jordi Bayona-i-Carrasco), y de desplazamientos residenciales y su impacto en la etnificación y en los alquileres (Juan Manuel Parreño-Castellano, Josefina Domínguez-Mujica y Claudio Moreno-Medina).

Por otro lado, un segundo grupo de tres artículos centran su atención en aspectos relativos a los cambios demográficos y en determinados colectivos, como los cambios residenciales y la composición de los hogares entre la población mayor (Cristina López Villanueva, Miguel Rubiales Pérez e Isabel PujadasRúbies), la gentrificación en las dos grandes ciudades españolas (Antonio López-Gay, Joan Sales-Favà, Miguel Solana Solana, Ana Fernández y Andrés Peralta), y en los cambios demográficos y la vulnerabilidad en municipios de los que emigra población joven (Alejandro López González).

Entre los primeros, el artículo de Olga de Cos (Cuando el periurbano cobra vida propia. La movilidad diaria y residencial como claves para caracterizar el área metropolitana de Santander), analiza la configuración actual del área metropolitana de Santander, tomando como punto de partida el área urbana funcional. La autora estudia la organización interna del área funcional y cuantifica los principales procesos que allí acontecen: artificialización, dispersión, discontinuidad y un crecimiento demográfico que se manifiesta en más zonas ocupadas con menor densidad. Los resultados permiten poner en valor los estudios geográficos sobre ocupación del suelo y movilidad en las áreas urbanas funcionales, así como la expansión urbana tendencial, fragmentadora y altamente consumidora de suelo. A partir de la movilidad diaria, la autora aborda la delimitación del área urbana funcional y del área metropolitana, concluyendo que ambas no tienen un reflejo espacial idéntico y no son conceptos sinónimos, lo cual tiene implicaciones importantes en clave de gobernanza. También se destaca la utilidad de las fuentes usadas en este trabajo, ya que permitirían analizar la cuestión metropolitana con un enfoque similar en otros sistemas españoles y, en su caso, europeos. 
El segundo artículo del primer grupo, el de Julia Salom-Carrasco (Cambios residenciales y migratorios de la población extranjera en la ciudad de Valencia en el periodo post-crisis, 2014-2017), se centra en cómo la llegada de población extranjera a la ciudad de Valencia ha influido en el paisaje social, dando lugar a concentraciones espaciales de este tipo de población en áreas concretas. La autora analiza la localización de la población extranjera en el área central y metropolitana, y los cambios residenciales y migratorios a escala de sección censal. Los resultados revelan que la movilidad residencial y migratoria de la población extranjera está contribuyendo a la consolidación y ampliación geográfica del patrón territorial existente, detectándose algunos cambios debidos a la emergencia de nuevos espacios atractivos para la población extranjera de nivel educativo más elevado, lo que se produce, especialmente, entre nacionales de países de la Unión Europea y en entornos específicos.

También Andreu Domingo y Jordi Bayona-i-Carrasco, a partir de la teoría del "metabolismo demográfico" (en su trabajo La huella de las migraciones en el metabolismo demográfico del Área Metropolitana de Barcelona), dan cuenta del cambio social a través de la sucesión de las diferentes generaciones en un territorio determinado. Con datos del Registro de Población de Cataluña y para el Área Metropolitana de Barcelona, los autores aplican esta perspectiva de forma retrospectiva para analizar la composición por género, generación y origen de las personas que viven en los diferentes barrios de la ciudad de Barcelona y en los distritos de su área metropolitana. Por un lado, este análisis ha permitido caracterizar el barrio en cuanto a la composición de la población por origen, sexo y edad. Por otro lado, el aumento polarizado de efectivos lleva asociado un crecimiento de la heterogeneidad intra e intergeneracional, y la convivencia (y segregación) de los residentes de cada área estudiada es interpretada como condición previa para el contacto intercultural teniendo en cuenta la edad, el sexo y el nivel de estudios, el lugar de nacimiento y la procedencia. Los autores anticipan que uno de los impactos de la COVID-19 en las zonas analizadas va a ser una aceleración del metabolismo demográfico debido a la incidencia de la mortalidad en las generaciones mayores pero, sobre todo, la circulación de vivienda que estos óbitos va a provocar, haciendo visible el factor de mercado que se oculta tras la substitución de las generaciones.

La última contribución del primer bloque es la realizada por Juan Manuel Parreño-Castellano, Josefi- na Domínguez-Mujica y Claudio Moreno-Medina (Etnificación, desahucio por impago de alquileres y desplazamiento residencial en una ciudad turística insular, Las Palmas de Gran Canaria - España). Se trata de un estudio geográfico de carácter demográfico y sociourbano acerca de la vulnerabilidad y las pautas de exclusión o segregación residencial de la población extranjera. Como consecuencia de la llegada de trabajadores y residentes de origen extranjero a Las Palmas de Gran Canaria, los espacios centrales de la ciudad han visto multiplicarse los desahucios por el impago de rentas. Ante este hecho, los autores analizan la relación espacial entre la presencia de población nacida en el extranjero y los procesos de desahucio por impago de alquileres entre 2008 y 2017 . También se estudia el desplazamiento residencial de este colectivo, que se produce en paralelo al incremento de los precios y lanzamientos de las viviendas en alquiler, indagando en los procesos de sustitución que conllevan según el lugar de origen de la población. La combinación de datos, obtenidos de las Estadísticas del Padrón Continuo y el Registro del Servicio Común de Notificaciones y Embargos de la ciudad, les ha permitido realizar un detallado análisis cartográfico y estadístico. Además de destacar el elevado número de extranjeros que reside en la ciudad, el esfuerzo de pago que deben realizar y el aumento de inseguridad residencial en las zonas centrales de la ciudad, los autores apuntan a la posible producción de un proceso de gentrificación. Este trabajo revela, como también el de Lopez Gay et al., dinámicas en las que interviene el modo de producción capitalista a través de la especialización turística y de las desigualdades sociales inherentes a su puesta en marcha.

El segundo grupo de artículos está integrado, primero, por el de Cristina López Villanueva, Miguel Rubiales Pérez e Isabel Pujadas-Rúbies (Transformaciones sociodemográficas, independencia residencial $y$ condiciones de vida en los hogares de los mayores en Barcelona). Estos autores sitúan en un primer plano el envejecimiento de la población y los cambios en los hogares de las personas de edad. Su objetivo es estudiar la composición de los hogares de los mayores en la ciudad de Barcelona y su relación con las características de la vivienda, las condiciones económicas y la percepción del estado de salud. Para ello, hacen una explotación de los microdatos de la Encuesta Sociodemográfica de Barcelona, realizada por el Ayuntamiento de Barcelona en 2017. En el artículo se analiza la reducción del tamaño del hogar y el aumento de la independencia residencial, que se manifiesta en una proporción creciente de mayores que comparten 
el hogar con su pareja o cónyuge o bien que residen en solitario, mientras que disminuye la proporción de personas que viven en hogares intergeneracionales. Los autores destacan la independencia residencial a edades cada vez más avanzadas, la mayor presencia de personas que viven solas y con menos familiares o allegados, y cómo las características socioeconómicas y habitacionales incrementan la fragilidad de los mayores. Los resultados llevan a destacar que factores sociodemográficos como la edad y el sexo, el estado de la vivienda, los recursos económicos y la red de apoyo y la salud, son elementos condicionantes de la vulnerabilidad de los mayores, apuntado así la relación existente entre diferentes dimensiones de vulnerabilidad en cada tipo de hogar. El trabajo pretende contribuir a la comprensión de la evolución de los tipos de hogar en la vejez y a aportar elementos de reflexión sobre la independencia residencial de los mayores en sus propios hogares y barrios, hacia los que muestran apego y arraigo. Concluyen señalando que las ciudades deben adaptarse a las poblaciones envejecidas con necesidades crecientes, tanto en lo que se refiere a la adecuación de los hogares, a los soportes y apoyos y a las infraestructuras de todos los elementos del entorno residencial.

El artículo de Antonio López-Gay, Joan Sales-Favà, Miguel Solana Solana, Ana Fernández y Andrés Peralta (El avance de la gentrificación en Barcelona y Madrid, 2011-2019: análisis socioespacial a partir de un índice de gentrificación) compara el proceso de gentrificación en dos grandes ciudades. Es un tema de actualidad y ampliamente tratado y debatido por parte de investigadores, administraciones públicas, entidades sociales y medios de comunicación. Por un lado, los autores calculan un índice de gentrificación para medir la intensidad de los procesos de transformación sociodemográfica en los barrios de Barcelona y Madrid desde 2011 hasta 2019; por otro, comparan los patrones espaciales del índice calculado y exploran las similitudes y singularidades con las que se ha manifestado la gentrificación a partir de la vulnerabilidad social de los barrios, del perfil de los nuevos habitantes, de la expulsión y desplazamiento de la población, y de la velocidad del cambio poblacional. Los resultados muestran la expansión de la gentrificación hacia áreas que habían permanecido ajenas a estas dinámicas, así como la existencia de rasgos distintivos en los procesos de ambas ciudades. Todavía se dispone de poca evidencia cuantitativa que permita diseccionar la diversidad de elementos sociodemográficos que intervienen en los procesos de gentrificación de las ciudades españolas. Sin embargo, esta investigación proporciona un índice de gran potencialidad como herramienta para la toma de decisiones, y de enorme recorrido para el diagnóstico de los procesos de transformación urbana y de la composición sociodemográfica. Su uso permitirá comparar la intensidad de los procesos en diferentes momentos y comprender la forma en la que interaccionan cada una de las dimensiones del índice de gentrificación en el tiempo y en el espacio.

Finalmente, el artículo de Alejandro López González (Vulnerabilidad demográfica y distancia a grandes ciudades: Consecuencias sobre la población adultojoven en Castilla y León) aborda los cambios demográficos y la vulnerabilidad en municipios en Castilla y León desde los que emigra población joven. Por su temática, es la única aportación que abandona el espacio urbano y metropolitano como objeto de estudio, variando la escala de análisis respecto a las anteriores contribuciones. Este trabajo plantea la hipótesis de una segunda despoblación, protagonizada por jóvenes residentes en ciudades de las regiones que forman la España interior. Tomando como área de estudio la Comunidad de Castilla y León, el autor diseña una metodología que parte de una primera clasificación de los municipios de esta región en función de su grado de vulnerabilidad demográfica. Asimismo, aplica un modelo autorregresivo espacial para medir el impacto sobre el porcentaje de población de edades entre 20 y 39 años de la distancia a Madrid y a Valladolid, considerando estas áreas como potenciales receptores de la población joven que emigra desde sus municipios de origen. Según sus resultados, la crisis demográfica está presente en todo el territorio regional y, aunque es mayor en los municipios rurales situados en áreas geográficamente marginales, también se ha extendido a territorios con mayores posibilidades de desarrollo como las ciudades y sus periferias. Los resultados apuntan a que el impacto de la emigración es mayor sobre aquellos municipios de carácter rural y sobre las cabeceras comarcales clásicas, pero no son tan claros sobre las cohortes adulto-jóvenes residentes en las ciudades medias ni en los municipios económicamente más dinámicos.

En su conjunto, los artículos de esta sección monográfica son de gran variedad y riqueza de objetivos, de fuentes y de técnicas analíticas, aunque se aplican básicamente metodologías cuantitativas. Las principales fuentes y más utilizadas son los Censos de la Población y de las Viviendas, el Padrón de Habitantes, los microdatos de la Estadística del Padrón Continuo y la Estadística de Variaciones Residenciales, procedentes del Ins- 
tituto Nacional de Estadística (INE). Otras fuentes son el Registro Estadístico de la Población ofrecido por el Instituto de Estadística de Catalunya (IDESCAT), los microdatos de la Encuesta Sociodemográfica de Barcelona (ESDB), e incluso alguna no estrictamente demográfica como los registros incluidos en el sistema de gestión procesal y los datos oficiales sobre lanzamientos que publica el Consejo General del Poder Judicial -todo ello para el análisis de los desahucios, en el trabajo sobre Las Palmas de Gran Canaria-. En la mayoría de trabajos se integran fuentes estadísticas y cartográficas diversas, considerando igualmente fuentes como el Global Human Settlement y las coberturas de Copernicus tratadas mediante SIG en el caso de la ciudad de Santander, de gran potencial para el estudio de los procesos de expansión urbana a nivel supramunicipal.

También la diversidad es grande en cuanto a las técnicas empleadas. En unos casos, se aplican herramientas estadísticas (I Global de Moran y Hot-Spot Analysis) para identificar las tendencias espaciales significativas, realizándose análisis de agrupamiento espacial. En otros trabajos se utilizan técnicas fundamentadas en la autocorrelación espacial, y la construcción de distintos índices como los de diversidad y de vulnerabilidad demográfica, y de gentrificación urbana, así como métodos de clasificación y agregación de municipios con identificación de clústeres. Todas estas fuentes, técnicas y métodos reflejan la solidez de los análisis y el grado de robustez con que, desde la disciplina geodemográfica, se afronta el estudio de los procesos y transformaciones sociodemográficas y territoriales producidos en los espacios urbanos en España.

Finalmente, y para concluir este marco de presentación, es preciso señalar que las aportaciones presentadas en el congreso (algunas publicadas en esta sección especial, y otras en la revista AGER: Revista de Estudios sobre Despoblación y Desarrollo Rural, y también en la Revista Internacional de Estudios Migratorios -RIEM-) ponen de manifiesto la buena salud y diversidad de la investigación en geografía de la población en España. De los trabajos que anteceden y su afrontamiento (planteamiento de objetivos, desarrollo y obtención de resultados) se considera que sólo el avance académico y científico en esta disciplina, desterrando las noticias falsas y alarmistas que en estos tiempos surgen con frecuencia, puede ayudar en la planificación territorial.

\section{REFERENCIAS}

González-Leonardo, M. (2020). Declive demográfico y envejecimiento en las capitales de provincia. Cuadernos Geográficos, 60(3), 168-191. doi: 10.30827/cuadgeo.v60i3.17719

López Gay, A. (2016). El advenimiento de las regiones metropolitanas maduras en España: retos demográficos y socioeconómicos en un nuevo contexto urbano. Panorama Social, (23), 79-196.

Módenes, J. A. y López-Colás, J. (2014). Cambio demográfico reciente y vivienda en España: ¿hacia un nuevo sistema residencial?. Revista Española de Investigaciones Sociológicas, (148), 103-134. doi:10.5477/cis/reis.148.103

Torrado, J. M., Calvache, R. D. y Susino, J. (2020). El retorno de la ciudad. La tesis de la recuperación material y simbólica de las ciudades centrales. $B i$ blio3W. Revista Bibliográfica de Geografía y Ciencias Sociales, XXV(1299), 1-36.

Torrado, J. M.; Duque-Calvache, R. y Palomares-Linares, I. (2020). Procesos de centralización urbana: factores individuales y tipologías metropolitanas. Documents d'Anàlisi Geogràfica, 66(3), 649-672. doi: $10.5565 / \mathrm{rev} /$ dag.568 